\title{
A COMPACT LED LIDAR SYSTEM FITTED FOR A MARS ROVER - DESIGN AND GROUND EXPERIMENT
}

\author{
Prane Mariel B. Ong ${ }^{1, *}$, Tatsuo Shiina ${ }^{1}$, Naohiro Manago ${ }^{1}$, Hiroaki Kuze ${ }^{1}$, Hiroki Senshu ${ }^{2}$, \\ Naohito Otobe ${ }^{3}$, George Hashimoto ${ }^{4}$, and Yasuhiro Kawabata ${ }^{5}$ \\ ${ }^{1}$ Chiba University, 1-33 Yayoi-cho, Inage-ku, Chiba, 263-8522, Japan, *Email: prane.ong@gmail.com \\ ${ }^{2}$ Chiba Institute of Technology, 2-17-1 Tsudanuma, Narashino-shi, 275-0016 Japan \\ ${ }^{3}$ Fukuoka University, 8-19-1 Nakakuma, Jonan-ku, Fukuoka, 814-0180 Japan \\ ${ }^{4}$ Okayama University, 1-1-1 Tsushima-naka, Kita-ku, Okayama, 700-8530 Japan \\ ${ }^{5}$ Meteorological Research Institute, 1-1 Nagamine, Tsukuba, Ibaraki, 305-0052 Japan
}

\begin{abstract}
A compact LED lidar was constructed and fieldtested with the aim to observe the Mars' dust devils. To be able to fit it on the Mars rover, a specialized Cassegrain telescope was designed to be within a $10 \mathrm{~cm}$-cube, with a field of view of $3 \mathrm{mrad}$. The transmitter has $385 \mathrm{~nm}$ LED light source with 3 $\mathrm{cm} \phi$ opening, $70 \mathrm{mrad}$ divergence, $0.75 \mathrm{~W}$ (7.5nJ/10ns) pulse power, and $500 \mathrm{kHz}$ repetition frequency. The configuration of the optical system is biaxial to easily configure the overlap between their optical axes.
\end{abstract}

\section{INTRODUCTION}

Mars explorations and missions have continuously been conducted for several decades now to monitor Mars temperature, biological life, atmosphere, and dust [1]. Although a considerable progress has been made on these, knowledge on the near-ground atmosphere is still lacking due to restricted ground observations [1-4].

One of particular interest is the "dust devil" or strong whirlwind, because of their influence in Martian climate, and how it can affect the rover exploration [5]. Although the motion and size of the observed Martian dust devils can be estimated from images obtained from satellites and rovers, still the quantitative information from these estimates were poorly constrained [6]. Hence, in situ measurements of near-ground atmospheric and dust activities on Mars will be useful in the study of this phenomenon.

Lidar (Light Detection and Ranging) may be able to measure the structure of whirlwinds along the line of sight. Lidar typically uses laser light to quantify the target's distance to the light source. However, a classical laser lidar system is generally too large in volume and in mass to be onboard a Mars rover, and it needs a cooler that requires large amount of power to be operational.

Given these restrictions and the extreme surface temperature condition on Mars, our team have examined the use of LED (light emitting diode) as the light source of the mini lidar system to be installed in a Mars rover. Aside from being energy efficient, heat sink independent, and tough against circumference change, previous researches on LED lidar have proved that the LED lidar can be used in near-ground Earth atmosphere monitoring [7-12]. The beam radiation angle is wide compared to its laser counterpart, but this wide beam divergence of the transmitter will establish a constant overlap in the near-field with the receiver's narrow field of view (F.O.V.). Hence, the system design has been focused on the robustness against tough treatment, transportability (10 cm-cube limit), and capability for near-range monitoring $(\sim<30 \mathrm{~m})$ of dust activities.

\section{SYSTEM DESIGN}

The goal of the design was to fit the LED lidar within a $10 \mathrm{~cm}$-cube limit. In order to accomplish this, a Cassegrain telescope was chosen as the receiver with specially designed primary mirror to shorten its barrel.

To date, there are two compact LED lidars that were fabricated: version 1 , and version 2 . Version 2 was the improved version of version 1 specifically in terms of compactness, design, and stability. Both of their receivers have the same optical and electrical parameters. The aperture and focal length were $10 \mathrm{~cm} \phi$ and $72.25 \mathrm{~mm}$, respectively. The $2.5 \mathrm{~cm} \phi$ diameter secondary mirror has a $-25 \mathrm{~mm}$ focal length. In both receivers, $45^{\circ}$ mirror, iris, interference filter, and 
photomultiplier tube (PMT) detector were incorporated in the rear space of the telescope.

On the other hand, the transmitter for both versions was almost identical except for the optics used. Essentially, the transmitter has a $385 \mathrm{~nm}$ wavelength NUV-LED, which is commercially manufactured by Nichia Corporation. It has a $0.75 \mathrm{~W}$ pulsed power with $10 \mathrm{~ns}$ pulse width at 500 $\mathrm{kHz}$ pulse-repetition frequency given a beam divergence of about $70 \mathrm{mrad}$. The transmitter opening is about $3 \mathrm{~cm} \phi$. A high pulse repetition frequency was set to improve the signal-to-noise ratio (SNR) due to the low pulse energy (7.5nJ/10ns). The pulse driver was specially designed to be separated from the transmitter's LED device (with optics unit), to protect it from the varying surface temperature of Mars. The transmitter and receiver parameters are summarized in Table 1.

To be able to follow the high repetition frequency $(500 \mathrm{kHz})$ of the transmitter, the photon counter was fabricated using a high-speed FPGA (Spartan 6 , Xilinx) with a time resolution as low as $1 \mathrm{~ns}$ or $0.15 \mathrm{~m}$ distance resolution equivalent. The power consumption of the current photon counter is $2 \mathrm{~W}$ for 1 channel DAQ, but it was verified that it can still be reduced to $1 \mathrm{~W}$.

To be able to perform the ground experiment and the initial performance evaluation, the compact LED lidar transmitter and receiver were housed in a polyvinyl chloride as the frame material (see Figure 1). The transmitter-receiver configuration for both LED lidars was arranged in a biaxial mode with an angle adjuster, so the overlap distance of the transmitter beam with the receiver's optical axis can easily be controlled. Moreover, during experiment, the LED lidar system was mounted in user-controlled tracker to easily adjust the altitude and azimuth directions.

\section{SYSTEM PERFORMANCE}

Currently, two experiments: wind tunnel experiment, and field experiment, have been done in order to assess the performance of the developed LED lidars. The wind tunnel experiment was conducted using version 1 of the LED lidar, and the field experiment was conducted using version 2 of the LED lidar.
Table 1 Parameters of the transmitter and the receiver.

\begin{tabular}{|r|c|}
\hline \multicolumn{2}{|c|}{ Transmitter } \\
\hline Light Source & $\begin{array}{l}\text { NUV-LED NCSU034B } \\
\text { (Nichia Corp.) }\end{array}$ \\
\hline Wavelength & $385 \mathrm{~nm}$ \\
\hline Pulse Width & $10 \mathrm{~ns}$ \\
\hline Pulsed Power & $0.75 \mathrm{~W}$ \\
\hline Pulse-Repetition Frequency & $500 \mathrm{kHz}$ \\
\hline Beam Divergence & $70 \mathrm{mrad}$ \\
\hline Beam Size & $3 \mathrm{~cm} \phi$ \\
\hline Cassegrain Receiver \\
\hline Barrel & $5 \mathrm{~cm}$ \\
\hline F.O.V. & $3 \mathrm{mrad}$ \\
\hline Primary Mirror Aperture & $10 \mathrm{~cm} \phi$ \\
\hline Primary Mirror Focal Length & $72.25 \mathrm{~mm}$ \\
\hline Secondary Mirror Diameter & $2.5 \mathrm{~cm} \phi$ \\
\hline Secondary Mirror Focal \\
Length
\end{tabular}

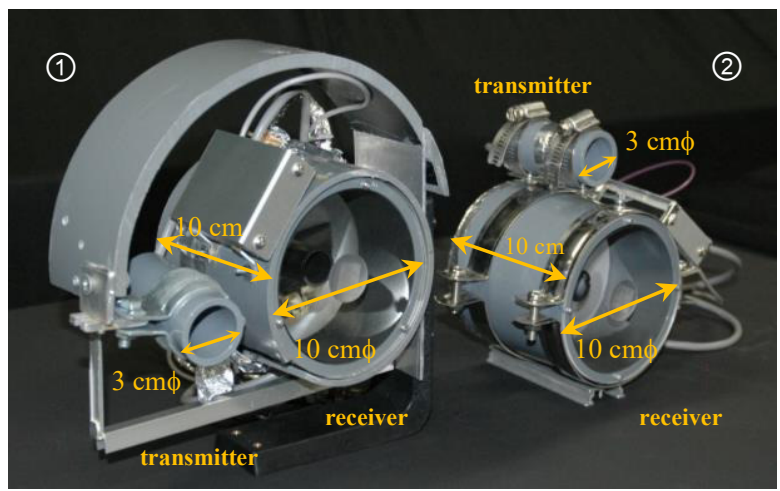

Figure 1 Two versions of the compact LED lidar for near-range ground experiment: (1) version 1, and

(2) version 2 .

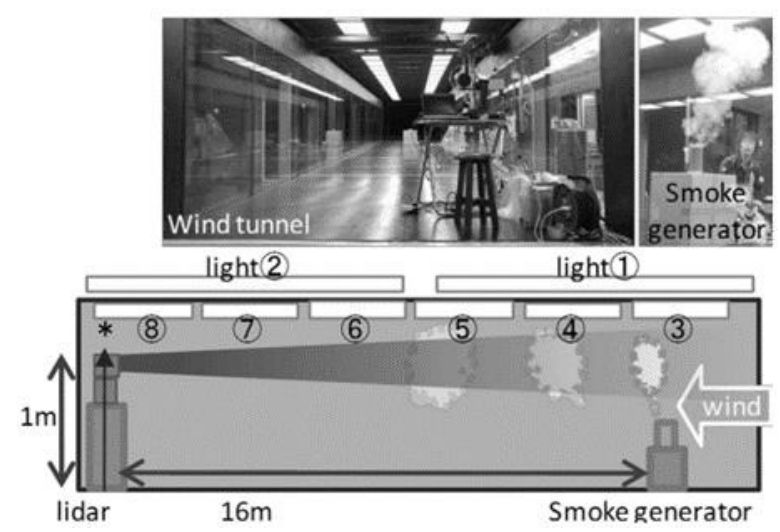

Figure 2 Setup for the wind tunnel experiment in the Japan Meteorological Agency wind tunnel facility. 


\subsection{Wind Tunnel Experiment}

This experiment was conducted in the wind tunnel facility of the Japan Meteorological Agency in Tsukuba, Japan. The LED lidar monitored the smoke's flow, diffusion and convection from a Glycerin smoke generator. The LED lidar and the Glycerin smoke generator arrangement is illustrated in Figure 2. The distance between them was set at $16 \mathrm{~m}$, where the wind direction is downwind with respect to the smoke generator, and the wind speed range is $0-5 \mathrm{~m} / \mathrm{s}$. Dust and particle counters were also installed near the smoke generator and were synchronized with the LED lidar measurements. The LED lidar was elevated 1 $\mathrm{m}$ from the ground to be vertically centered, since the ceiling height is $2 \mathrm{~m}$.

The backscattered smoke signal coming from the upward-pointing smoke generator with $20 \mathrm{~s}$ generation time interval is presented in Figure 3. A distance vs time graph with echo count as the value of the colormap is presented to analyze the smoke's flow over time. Given the smoke interval of $20 \mathrm{~s}$ and the wind speed of $1 \mathrm{~m} / \mathrm{s}$, it can be seen that at 14-15 $\mathrm{m}$ the LED lidar detected a strong backscattered echo from the smoke and gradually decreased overtime, then registered a slight increase after $10 \mathrm{~s}$. This illustrates the diffusion and convection of the smoke. Since we know that the smoke generator was pointing upward, this suggests that it might be the cause why the smoke initially rise for a few seconds before it transitioned to a lower ground. As the smoke diffused overtime in space and rose again in the LED lidar's line of sight due to convection, the measured backscatter smoke signal after $10 \mathrm{~s}$ was low compared to the initial reading at $14 \mathrm{~m}$.

We have also measured the backscattered signal from smoke with varying wind speeds: $0.3 \mathrm{~m} / \mathrm{s}, 0.5$ $\mathrm{m} / \mathrm{s}$, and smoke generator direction (directed towards the LED lidar). And observed that a high wind speed cuts down the smoke convection to a shorter period.

\subsection{Field Experiment}

In one of the 2 field experiments, the LED lidar was tested first by pointing to a hard target like a building. It was pointed to a building 50 meters away from the source. Figure 3 shows the hard target return from that building. The LED lidar was

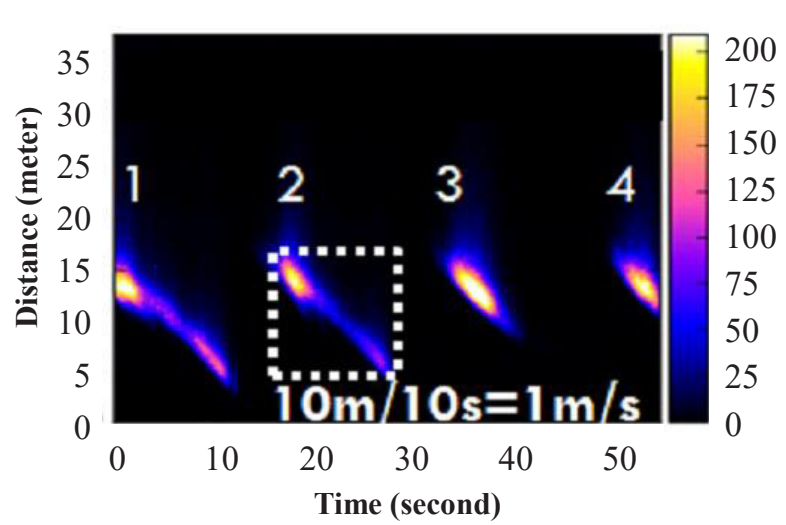

Figure 3 Smoke profile given the wind speed of $1 \mathrm{~m} / \mathrm{s}$ and smoke interval of $20 \mathrm{~s}$ using the LED lidar ver. 1 (Japan Meteorological Agency, Tsukuba, Japan).

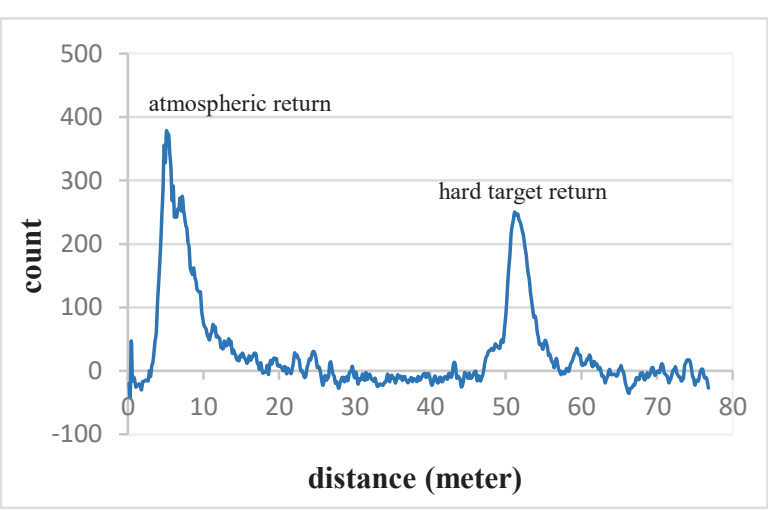

Figure 4 Hard target echo by the LED lidar ver. 2.

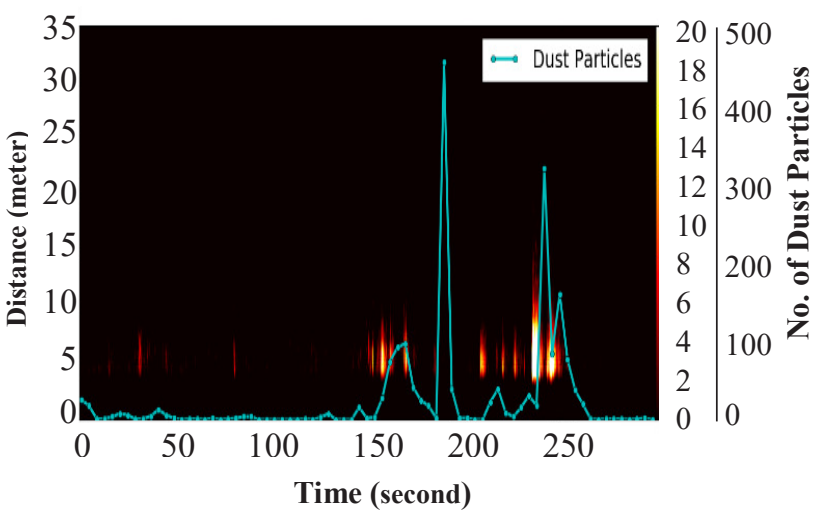

Figure 5 Dust profile with both LED lidar data (ver. 2) and dust counter data measured in InageKaigan field experiment (Chiba, Japan).

able to measure the atmospheric echo a few meters away from the system's receiver, because of the overlap between the beam's divergence and receiver's F.O.V., and the hard target return 
$(\sim 50 \mathrm{~m})$ for an accumulation time of $2 \mathrm{~s}$ with distance resolution of $0.15 \mathrm{~m}$ or time resolution of $1 \mathrm{~ns}$. The detectable range for the atmospheric return was about $30 \mathrm{~m}$.

The next field experiment was conducted in InageKaigan coast area in Chiba, Japan $\left(35^{\circ} 36^{\prime} 55.6^{\prime \prime} \mathrm{N}\right.$, $\left.140^{\circ} 03^{\prime} 48.1^{\prime \prime E}\right)$ on December 6, 2016. The LED lidar was mounted in a tracker to easily configure the elevation and azimuth directions. The system was approximately $30 \mathrm{~cm}$ above the ground, with $210^{\circ}$ azimuth and $2^{\circ}$ elevation angles. The wind direction is downwind with respect to the LED lidar system. At $30 \mathrm{~m}$ directly away from the system, we have also installed the anemometer, dust, and particle counters.

The LED lidar was set to a $4 \mathrm{~Hz}$ of sampling rate and a distance resolution of $1 \mathrm{~ns}$ or $0.15 \mathrm{~m}$ distance resolution, giving a total detectable range of 76.8 meters.

Figure 5 presents the distance vs time profile of the dust echo overlayed with dust particles measurement. During this time, the average wind speed is $6 \mathrm{~m} / \mathrm{s}$. As can be seen from the figure, there is a $5 \mathrm{~s}$ delay in the registered dust counter measurement compared to the LED lidar measurement. This is expected given that the wind direction originates at $340^{\circ}(\mathrm{NNW})$, which came from the rear of the LED lidar and towards the dust counter, which is $30 \mathrm{~m}$ away. At $245 \mathrm{~s}$, a $10 \mathrm{~m}$ dust echo was registered, which was the longest observed dust return, given the observation range of $35 \mathrm{~m}$.

\section{SUMMARY}

Two compact LED lidars were fabricated to fit the required $10 \mathrm{~cm}$-cube dimension. Version 2 has almost the same parameters (electronics and optics) as version 1 , but with more improved design and compactness for stability. They were both tested, although in two different environments. Both have shown promising results in near-range dust echo measurement. Although, the measurement is still restricted to nighttime. The lidar development is still in progress to be able to conduct field experiment during daytime.

\section{ACKNOWLEDGEMENT}

This project was supported by the JSPS KAKENHI grant (JP16H03129).

\section{Reference}

[1] Garber, S., 2015, April 16: A chronology of Mars exploration, Retrieved from http://history.nasa.gov/marschro.htm.

[2] Guzewich, S.D., Abshire , J. B., Smith, M.D., Riris, H., Sun, X., Gentry, B.M., Yu, A., and Allan,G. R., 2016: MARLI: MARs LIdar for global wind profiles from orbit, Proceedings of the 47th Lunar and Planetary Science Conference, $1497 \mathrm{pp}$.

[3] Whiteway, J., Daly, M., Carswell, A., Duck, T., Dickinson, C., Komguem, L., and Cook, C., 2008: Lidar on the Phoenix mission to Mars, J. Geophys. Res. 113, p. E00A08, doi:10.1029/2007/JE003002.

[4] Komguem, L., Whiteway, J. A., Dickinson, C., Daly, M., and Lemmon, M. T., 2013: Phoenix LIDAR measurements of Mars atmospheric dust, Icarus 223, 649-653pp.

[5] Jenner, L., 2007, November 30: Phantoms from the sand: tracking dust devils across Earth and Mars, Retrieved from https://www.nasa.gov/ vision/universe/solarsystem/2005_dust_devil.ht $\mathrm{ml}$.

[6] Ferri, F., Smith, P. H., Lemmon, M., and Rennó, N. O., 2003: Dust devils as observed by Mars Pathfinder, J. Geophys. Res. 108, 5133pp. doi:10.1029/2000JE001421.

[7] Koyama, M. and Shiina, T., 2010: Light Source Module for LED Lidar, Proceedings of $25^{\text {th }}$ International Laser Radar Conference, S01P32-1-4.

[8] Grishin M. Eds., 2010: Advances in Solid-State Lasers: Development and Applications, Chapter 8, INTECH.

[9] Fukuchi, T., and Shiina, T., Eds., 2012: Industrial Applications of Laser Remote Sensing, Bentham Science Publishers Ltd.

[10] Korotcenkov, G., Ed., 2013: Chemical Sensors Volume 4: Chapter 12, Optical Sensors.

[11] Shiina, T., Noguchi, K., and Tsuji, K., 2016: Compact and mini Raman lidars for hydrogen gas detection, Proceeding of ICONE-23, ICONE23-1640.

[12] Shiina, T., Yamada, S., Senshu, H., Otobe, N., Hashimoto, G. L., and Kawabata, Y., 2016: LED minilidar for Mars rover, Proc. SPIE 10006, 10006-15 pp. 